\title{
STABLE MAPS INTO THE HILBERT CUBE
}

\author{
DENNIS J. GARITY AND DALE M. ROHM
}

(Communicated by Doug W. Curtis)

\begin{abstract}
A map into the Hilbert cube is stable if each composition with projection onto a finite number of factors is stable. We prove that a map from a compact metric space into the Hilbert cube is stable if and only if it is universal. As a consequence, the composition of a stable map with any self homeomorphism of the Hilbert cube is also stable.
\end{abstract}

1. Introduction. All spaces are separable metric spaces. A map $f: X \rightarrow I^{n}$ is said to be stable if there does not exist a map $g: X \rightarrow S^{n-1}$ with $\left.f\right|_{f^{-1}\left(S^{n-1}\right)}=$ $\left.g\right|_{f^{-1}\left(S^{n-1}\right)}$. Stable maps are also known as Alexander-Hopf essential maps [N, GT]. Krasinkiewicz has given a general definition of essential maps into the product of manifolds $[\mathbf{K}]$ that coincides with the definition of stable maps in the cases under consideration. It is well known that a space has covering dimension greater than or equal to $n$ if and only if it admits a stable map into $I^{n}[\mathbf{H W}]$. Note that if $f: X \rightarrow I^{n}$ is a stable map and $h$ is any self-homeomorphism of $I^{n}$, then the composition $h \circ f$ is also stable, since $h\left(S^{n-1}\right)=S^{n-1}$.

Let $I^{\infty}=\prod_{i=1}^{\infty}[-1,1]_{i}$ denote the Hilbert cube, and for each $n$ let $p_{n}: I^{\infty} \rightarrow I^{n}$ be the projection map onto the first $n$ factors. The subsets $A_{n}=\left\{\left(x_{i}\right) \in I^{\infty} \mid x_{n}=\right.$ $-1\}$ and $B_{n}=\left\{\left(x_{i}\right) \in I^{\infty} \mid x_{n}=1\right\}$ are referred to as faces of the Hilbert cube. A map $f: X \rightarrow I^{\infty}$ from a compact metric space into the Hilbert cube is said to be stable if the composition $p_{n} \circ f: X \rightarrow I^{n}$ is stable for each $n$. See [W and $\mathbf{B}$ ] for a more detailed description of stable maps. In particular, Walsh showed that a map $f: X \rightarrow I^{\infty}$ from a compact metric space is stable if and only if the collection of pairs $\left\{\left(f^{-1}\left(A_{i}\right), f^{-1}\left(B_{i}\right)\right) \mid i=1,2, \ldots\right\}$ is an essential family for $X$, i.e., for any sequence of separators $\left\{S_{i}\right\}$ of $f^{-1}\left(A_{i}\right)$ and $f^{-1}\left(B_{i}\right), \bigcap_{i=1}^{\infty} S_{i} \neq \varnothing$. It follows that a compact metric space admits a stable map into the Hilbert cube if and only if it is strongly infinite dimensional.

Our goal is to prove a result for stable maps into the Hilbert cube which is analogous to the result noted above for stable maps into $n$-cells. Namely, we show that if $f: X \rightarrow I^{\infty}$ is stable and $h$ is any self-homeomorphism of $I^{\infty}$, then the composition $h \circ f$ is also stable. This gives a partial answer to a question of $\mathrm{J}$. Krasinkiewicz [K, Problem 1]. What we need for the proof is a characterization of stable maps in terms of a property preserved by self-homeomorphisms. Universality, a concept introduced by Holsztyński [H1] and widely used in the study of fixed point theory (see [H2, H3, H4, H5, H6, H7, GT and N]), is such a property. A map

Received by the editors July 21, 1987 and, in revised form, September 9, 1987.

1980 Mathematics Subject Classification (1985 Revision). Primary 54F45, 57N20; Secondary $54 \mathrm{H} 25,54 \mathrm{C} 10$.

Key words and phrases. Stable map, essential map, universal map, essential family, strongly infinite dimensional. 
$f: X \rightarrow Y$ is said to be universal if for every map $g: X \rightarrow Y$ there exists a point $p$ in $X$ with $f(p)=g(p)$. We will show that a map from a compact metric space into the Hilbert cube is stable if and only if it is universal. Then the desired result on preservation of stability by compositions with self-homeomorphisms of $I^{\infty}$ is an immediate corollary.

We would like to thank Doug W. Curtis for his helpful suggestions on the organization of this paper.

2. Universal maps and stable maps. The following result is contained in $[\mathbf{G T}, \mathbf{N}]$ and implicitly in [H1]. For completeness, we include a short proof.

THEOREM 1. Let $n$ be in $\mathbf{Z}_{+} \cdot A$ map $f: X \rightarrow I^{n}$ is stable if and only if it is universal.

ProOF. Suppose that $f: X \rightarrow I^{n}$ is a stable map. If $f$ were not universal, then we could find a map $g: X \rightarrow I^{n}$ so that $g(p) \neq f(p)$ for every point $p$ in $X$. Consider $S^{n-1}$ as the boundary of $I^{n}$ in the usual manner, and define $h: X \rightarrow S^{n-1}$ by setting $h(p)$ equal to the intersection of the ray containing $f(p)$ which emanates from $g(p)$ and $S^{n-1}$. Clearly $h$ is continuous and agrees with $f$ on $f^{-1}\left(S^{n-1}\right)$, contradicting the stability of $f$. Therefore, $f$ must be universal.

For the converse, suppose that $f: X \rightarrow I^{n}$ is universal and again consider $S^{n-1}$ as the boundary of $I^{n}$. If $f$ were not stable, then we could find a map $g: X \rightarrow S^{n-1}$ which agrees with $f$ on $f^{-1}\left(S^{n-1}\right)$. Composing with the antipodal map $\alpha: S^{n-1} \rightarrow$ $S^{n-1}$ would then give a map $\alpha \circ g: X \rightarrow S^{n-1} \subset I^{n}$ so that $\alpha \circ g(p) \neq f(p)$ for any point $p$ in $X$, contradicting the universality of $f$. Thus, $f$ must be stable.

The next result is the tool needed to link stability and universality of maps of compacta into the Hilbert cube.

THEOREM 2. A map $f: X \rightarrow I^{\infty}$ from a compact metric space into the Hilbert cube is universal if and only if each composition $p_{n} \circ f: X \rightarrow I^{n}$ is universal.

Proof. Assume that $f$ is universal. Fix a positive integer $n$. Consider the Hilbert cube as $I^{n} \times \prod_{j>n}[-1,1]_{j}$, and let a map $g: X \rightarrow I^{n}$ be given. By choosing a point $y_{j}$ in $[-1,1]_{j}$ for each $j>n$, we may assume that $g$ is a map into the Hilbert cube. Thus, since $f$ is universal, there exist a point $p$ in $X$ so that $g(p) \times \prod_{j>n}\left\{y_{j}\right\}=$ $f(p)$. Thus, $g(p)=p_{n} \circ f(p)$, and $p_{n} \circ f$ is shown to be universal.

For the converse, we assume that the Hilbert cube has the metric given by $d\left(y, y^{\prime}\right)=\sum_{i=1}^{\infty}\left(\left|y_{i}-y_{i}^{\prime}\right| / 2^{i}\right)$. Suppose that $p_{n} \circ f$ is universal for each positive integer $n$. If $f$ were not universal, then there exists a map $g: X \rightarrow I^{\infty}$ so that for every point $p$ in $X, g(p) \neq f(p)$. By the compactness of $X$, there exists a number $\delta>0$ so that for every point $p$ in $X$ the distance $d(g(p), f(p))>\delta$. But we can choose a positive integer $N$ so that the $\operatorname{diam}\left(\prod_{i=N+1}^{\infty}[-1,1]_{i}\right)<\delta$. This would imply that, for any point $p$ in $X, p_{N} \circ g(p) \neq p_{N} \circ f(p)$ contradicting the universality of $p_{N} \circ f(p)$. Therefore, $f$ must be universal.

We are now ready to obtain the results mentioned at the end of the previous section.

COROllary 1. A map $f: X \rightarrow I^{\infty}$ from a compact metric space into the Hilbert cube is stable if and only if it is universal.

PROOF. This is immediate from Theorems 1 and 2. 
COROLlaRY 2. If $f: X \rightarrow I^{\infty}$ is stable and $h$ is a self-homeomorphism of $I^{\infty}$, then $h \circ f$ is also stable.

PrOOF. Clearly, universality of maps is preserved by composition with selfhomeomorphisms of $I^{\infty}$. By Corollary 1, the same is true for stable maps.

COROLlaRY 3. Let $\left\{\left(C_{i}, D_{i}\right)\right\}_{i=1}^{\infty}$ be an essential family for a strongly infinitedimensional compact space $X$, and let $f$ be a map from $X$ into $I^{\infty}$ so that $C_{i}=$ $f^{-1}\left(A_{i}\right)$ and so that $D_{i}=f^{-1}\left(B_{i}\right)$ for each $i$. If $h$ is any self-homeomorphism of $I^{\infty}$, then $\left\{\left((h \circ f)^{-1}\left(A_{i}\right),(h \circ f)^{-1}\left(B_{i}\right)\right)\right\}_{i=1}^{\infty}$ is also an essential family for $X$.

PROOF. This follows immediately from Corollary 2 and Walsh's characterization of stability mentioned in $\S 1$.

\section{REFERENCES}

[B] P. L. Bowers, Detecting cohomologically stable mappings, Proc. Amer. Math. Soc. 86 (1982), 679-684.

[GT] J. Grispolakis and E. D. Tymchatyn, On confluent mappings and essential mappings--a survey, Rocky Mountain J. Math. 11 (1981), 131-153.

[H1] W. Holsztyński, Une généralisation du théorème de Brouwer sur les points invariants, Bull. Acad. Polon. Sci. Sér Sci. Math. Astronom. Phys. 12 (1964), 603-606.

[H2] _ Universal mappings and fixed point theorems, Bull. Acad. Polon. Sci. Sér. Sci. Math. Astrom. Phys. 15 (1967), 433-438.

[H3] _ A remark on the universcl mappings of 1-dimensional continua, Bull. Acad. Polon. Sci. Sér Sci. Math. Astrom. Phys. 15 (1967), 547-549.

[H4] _ Universality of mappings unto the products of snake-like spaces. Relation with dimension, Bull. Acad. Polon. Sci. Sér. Sci. Math. Astronom. Phys. 16 (1968), 161-167.

[H5] _ Universality of the product mappings onto the product of $I^{n}$ and snake-like spaces, Fund. Math. 64 (1969), 147-155.

[H6] _- On the composition and products of universal mappings, Fund. Math. 64 (1969), 181-188.

[H7] $\_$, On the product and composition of universal mappings of manifolds into cubes, Proc. Amer. Math. Soc. 58 (1976), 311-314.

[HW] W. Hurewicz and H. Wallman, Dimension theory, Princeton Univ. Press, Princeton, N.J., 1941.

[K] J. Krasinkiewicz, Essential mappings onto products of manifolds, preprint.

[N] S. B. Nadler, Universal mappings and weakly confluent mappings, Fund. Math. 110 (1980), 221-235.

[W] J. J. Walsh, A class of spaces with infinite-cohomological dimension, Michigan Math. J. 27 (1980), 215-222.

\footnotetext{
Department of Mathematics, Oregon State University, Corvallis, Oregon 97331

Department of Mathematics, Vanderbilt University, NashVille, Teinnessee 37235
} 